\title{
Low Power and High Reliable Triple Modular Redundancy Latch for Single and Multi-node Upset Mitigation
}

\author{
S Satheesh Kumar ${ }^{1}$, S Kumaravel ${ }^{2}$ \\ School of Electronics Engineering, VIT University, Vellore, India
}

\begin{abstract}
CMOS based circuits are more susceptible to the radiation environment as the critical charge $\left(Q_{\text {crit }}\right)$ decreases with technology scaling. A single ionizing radiation particle is more likely to upset the sensitive nodes of the circuit and causes Single Event Upset (SEU). Subsequently, hardening latches to transient faults at control inputs due to either single or multi-nodes is progressively important. This paper proposes a Fully Robust Triple Modular Redundancy (FRTMR) latch. In FRTMR latch, a novel majority voter circuit is proposed with a minimum number of sensitive nodes. It is highly immune to single and multi-node upsets. The proposed latch is implemented using CMOS $45 \mathrm{~nm}$ process and is simulated in cadence spectre environment. Results demonstrate that the proposed latch achieves $17.83 \%$ low power and $13.88 \%$ low area compared to existing Triple Modular Redundant (TMR) latch. The current induced due to transient fault occurrence at various sensitive nodes are exhibited with a double exponential current source for circuit simulation with a minimum threshold current value of 40 $\boldsymbol{\mu A}$.
\end{abstract}

Keywords-Multiple Event Transient (MET); Single Event Upset (SEU); Single Event Transient (SET); Radiation hardening; Reliability; Transient fault; Triple Modular Redundancy (TMR)

\section{INTRODUCTION}

The reliability issues are a major concern in semiconductor ICs designed for medical, space and defense applications that operate in a high radiation environment. As the CMOS technology scaling down, the supply voltage and node capacitance scales down as well. When high energy neutron or alpha particles passes through the MOS device, it causes an additional charge (excess electron-hole pair) induced in the substrate [1]. In specific, the sensitive node is the drain terminal of the OFF transistor. The induced additional charge collects by the drain terminal and turns on the device, causing in a voltage transient (or glitch) at the output. These glitches are called transient faults and are temporary in nature. Modelling the effect of transient fault on both NMOS and PMOS is shown in Fig. 1. To model the impact on NMOS transistor the double exponential current source is connected between the drain and source terminals of NMOS transistor as shown in Fig. 1(a). If the transient fault occurs on the drain terminal of NMOS transistor, a negative current spike is generated [22]. If the input gate voltage, $\mathrm{V}_{\mathrm{G}}=0$ at that moment, $\mathrm{V}_{\mathrm{GD}}$ becomes greater than the threshold voltage $\left(\mathrm{V}_{\mathrm{TN}}\right)$, i.e. $\mathrm{V}_{\mathrm{GD}}>\mathrm{V}_{\mathrm{TN}}$ which runs the transistor in triode region. The output node then pulls down to logic 0 .
To model the impact on PMOS transistor, the double exponential current source is connected as shown in Fig. 1(b). If the transient fault occurs on the drain terminal of PMOS transistor, positive current spike is generated. As a result the output node pulls up to logic 1 . The output node recovers by removal of the current source [2]-[4]. If this transient pulse is propagated through memory element then, the Single Event Upset (SEU) occurs.

To evade these radiation effects, many hardened by design techniques have been proposed to deal with Single Event Transients (SETs), SEUs and Multiple Event Transients (METs) [5]-[21]. The advantages of these methods are that they are highly resistant to SETs and SEUs. The cost in terms of power dissipation, delay and area consumption for protecting memory elements from an SEU is substantial. SETs and SEUs are more general observed errors whereas, METs may occur due to packing density or single event triggering multiple transients.

This paper suggests a low power, less area and fully robust triple modular redundancy latch design (named as FRTMR) latch. The suggested latch is less sensitive to SEU and Multiple Event Upsets (MEU). The FRTMR latch comprises of three identical latch structures and a novel majority voter circuit. The three identical latch structures consist of six feedback loops (three of them will be active when the output of inverters INV1, INV2 and INV3 are 0 and the other three will be active when the output is 1). This saves power dissipation. The majority voter circuit designed with less number of transistors and less sensitive nodes compared to the existing classical TMR latch used in [12]. With less number of sensitive nodes, the probability of affecting the circuit due to transient faults is also less. FRTMR consumes considerably low power and less area than the existing classical TMR latch because of the less number of transistors used in majority voter circuit. Detailed analysis of FRTMR latch is presented in Section III.

The remaining sections of the paper are organized as follows: Section II discussed about some existing hardened latches. Section III describes the implementation, detailed analysis of proposed FRTMR latch with and without transient faults. Performance comparisons with existing latch are reported in Section IV. Section V concludes the paper. 


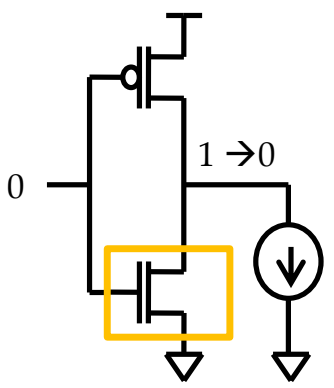

(a)

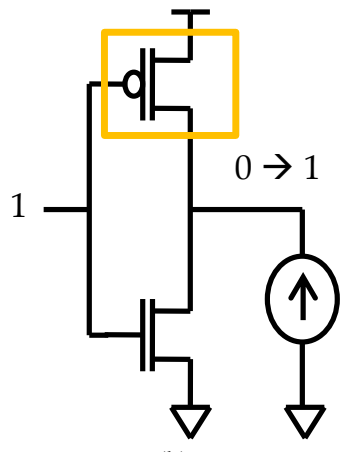

(b)
Fig. 1. Transient Fault Current Model on: (a) NMOS; (b) PMOS

\section{RELATED WORK}

Fig. 2 shows the conventional D-latch. This latch cannot function properly in highly radiated environments. During the latch mode, if a high energy particle strikes on the intermediates nodes $\mathrm{n} 1$ or $\mathrm{n} 2$. It may disrupt the state of the latch. This results in a wrong value at the output. To overcome this problem, many radiation-hardening latches have been proposed in the literatures.

Fig. 3 presents the circuit of the LCHR latch in [7]. The latch includes three redundant information retention feedback loops, which allows SEU to be tolerated in hold mode. The latch is capable of filtering single event transients arrived at input due to hysteresis property of Schmitt trigger (ST) inverter. Nevertheless, this circuit has following disadvantages: 1) in hold mode, there is a possible current competition, because the result is driven by a feedback loop and a Celement. This leads to more power dissipation. 2) Not economical because of area overhead and power dissipation. 3) Not completely self-recoverable from SEUs like DICE latch.

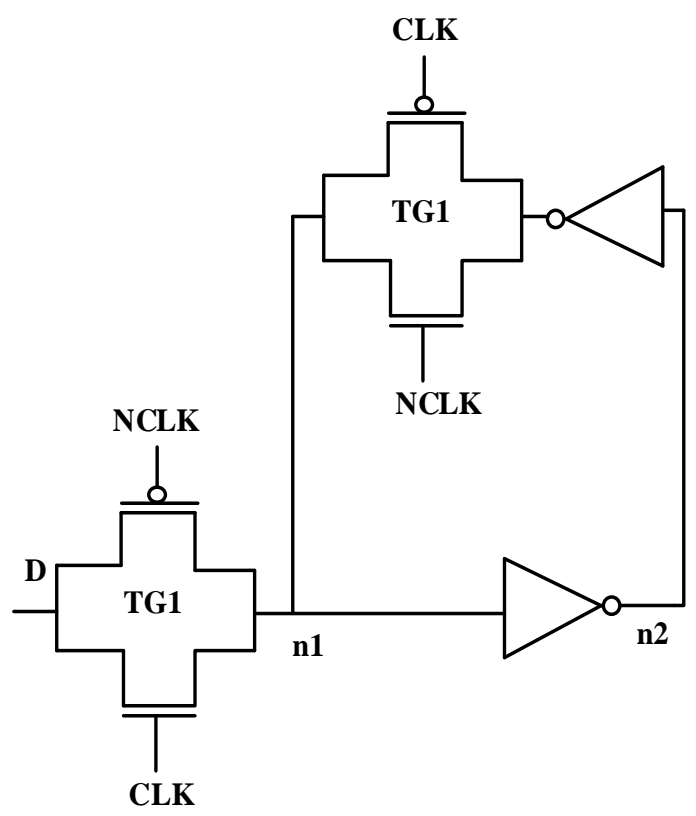

Fig. 2. Conventional Latch Structure.

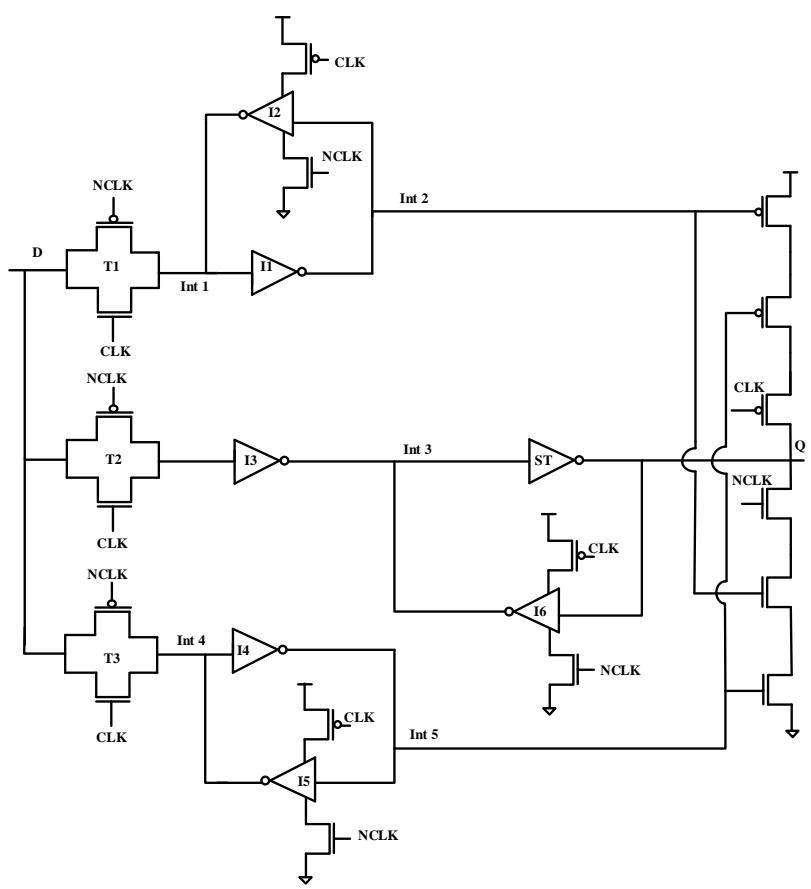

Fig. 3. LCHR Latch in [7].

The SEU resilient and SET filterable latch (RFEL) in [8] is presented in Fig. 4. This latch also includes three redundant information retention feedback loops in order to handle single node upsets due to particle strikes. The circuit uses a ST inverter in order to filter SETs in transparent mode of operation. But, the ST inverters cannot tolerate high energy particle strikes.

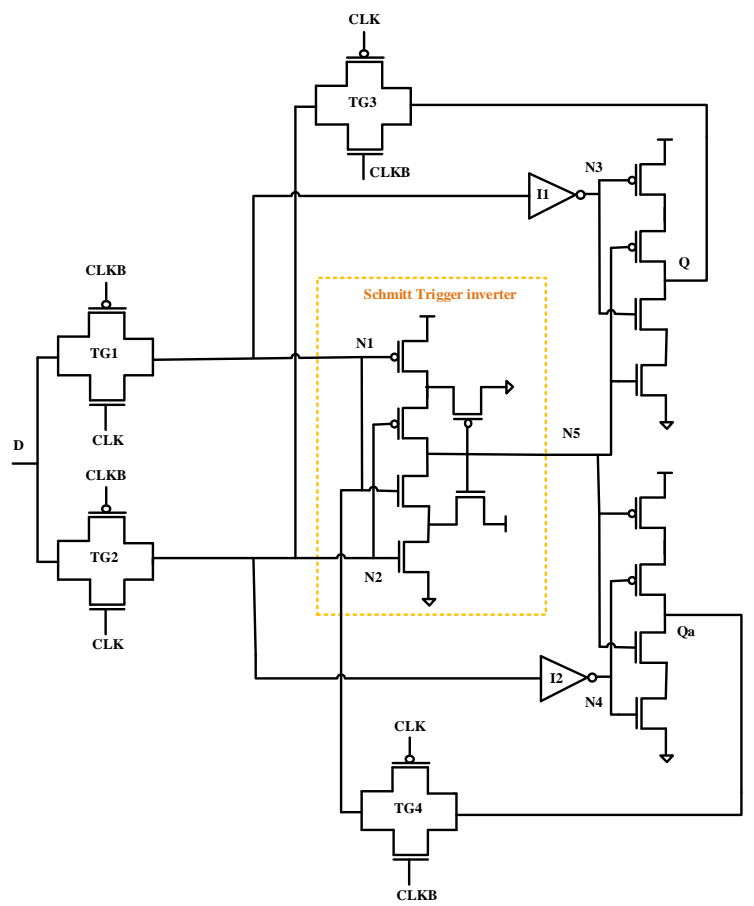

Fig. 4. RFEL Latch in [8]. 
Fig. 5 illustrates the latch circuit in [9]. The latch comprises of two interconnected structures. These cross-coupled structures form a negative feedback path. Feedback path for the latch are cut-off in transparent mode to improve the performance in terms of speed. In the latch mode, feedback structure enables to restore temporary failures because of SEUs. However, it cannot tolerate high energy particle strikes, and also consumes large silicon area.
The latch design in [10] is shown in Fig. 6. It comprises of a static D-latch and an error detection circuit. Error detection circuit plays a major role in mitigating soft errors. However, if the transient fault occurs on error detection circuit, it produces an erroneous value. Also, it has an extensive area overhead and power dissipation.

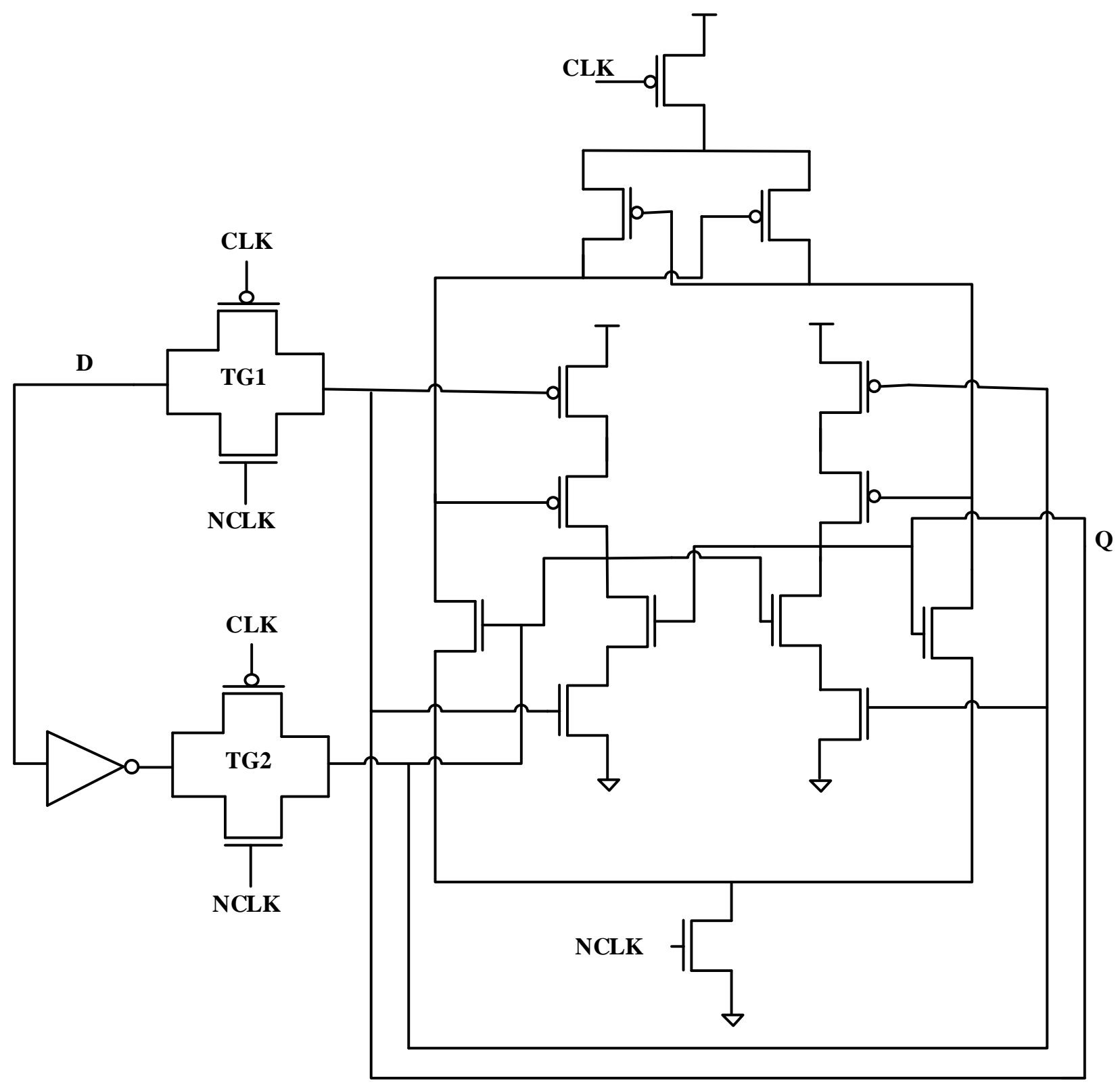

Fig. 5. Latch in [9]. 

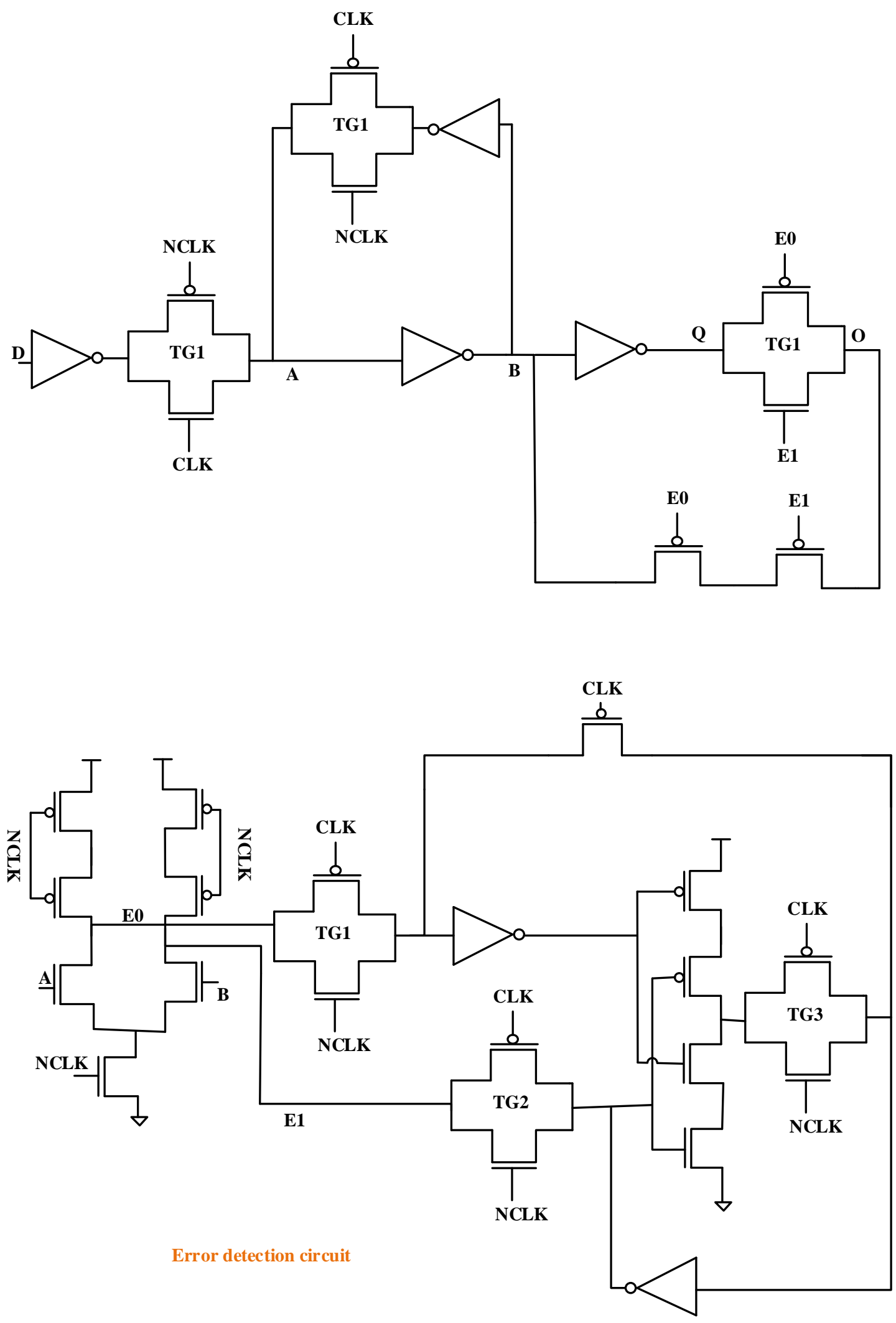

Fig. 6. Latch in [10]. 
The latch designed in [11] is shown in Fig. 7. It consists of three transmission gates, a memory element and a clocked Muller C-element to save power dissipation. Nonetheless, it cannot tolerate multi-node upsets and also if the transient fault occurs on intermediate nodes of $\mathrm{C}$-element it produces a glitch at the output. SEU tolerant latches to mitigate the single event upsets also include DICE, Quatro [20] and TMR latch used in $[12,13]$. All the above discussed latches are not multi-node upset tolerant.

The classical TMR latch is presented in Fig. 8. It consists of three identical latch circuits which individually perform the same operation and these results are processed by a majority voter circuit to produce a solitary output. It can tolerate SEU occurring on any one of the three identical latches, as long as the other two inputs continue to be stable, and provide $100 \%$ SEU immunity on the internal nodes N1 (or A), N2 (or B) and N3 (or C). In this paper these nodes are signified as "self- recoverable" (SR) nodes as the output can be self-recovered from any type of single node upsets. Nevertheless, an SET on the internal nodes $(\mathrm{n} 1-\mathrm{n} 5)$ of a voter circuit can cause a voltage transient at the output. These nodes are named as "critical nodes" (CN) as the output can't be recovered themselves until and unless the transient pulse is removed from the critical nodes.

Though, TMR latch is most prevalent hardened technique used in aerospace applications for its high reliability, many researchers tend to develop radiation hardened latch designs using the following techniques: 1) ST inverter. 2) Muller Celement. 3) DICE principle. 4) Error correction mechanism. The primary reason is that it incurs huge area and power dissipation. Also, the number of critical nodes is more. In addition to this, the existing TMR latch is not suitable for multi- node upsets. Our proposed FRTMR latch in section III resolves these issues properly.

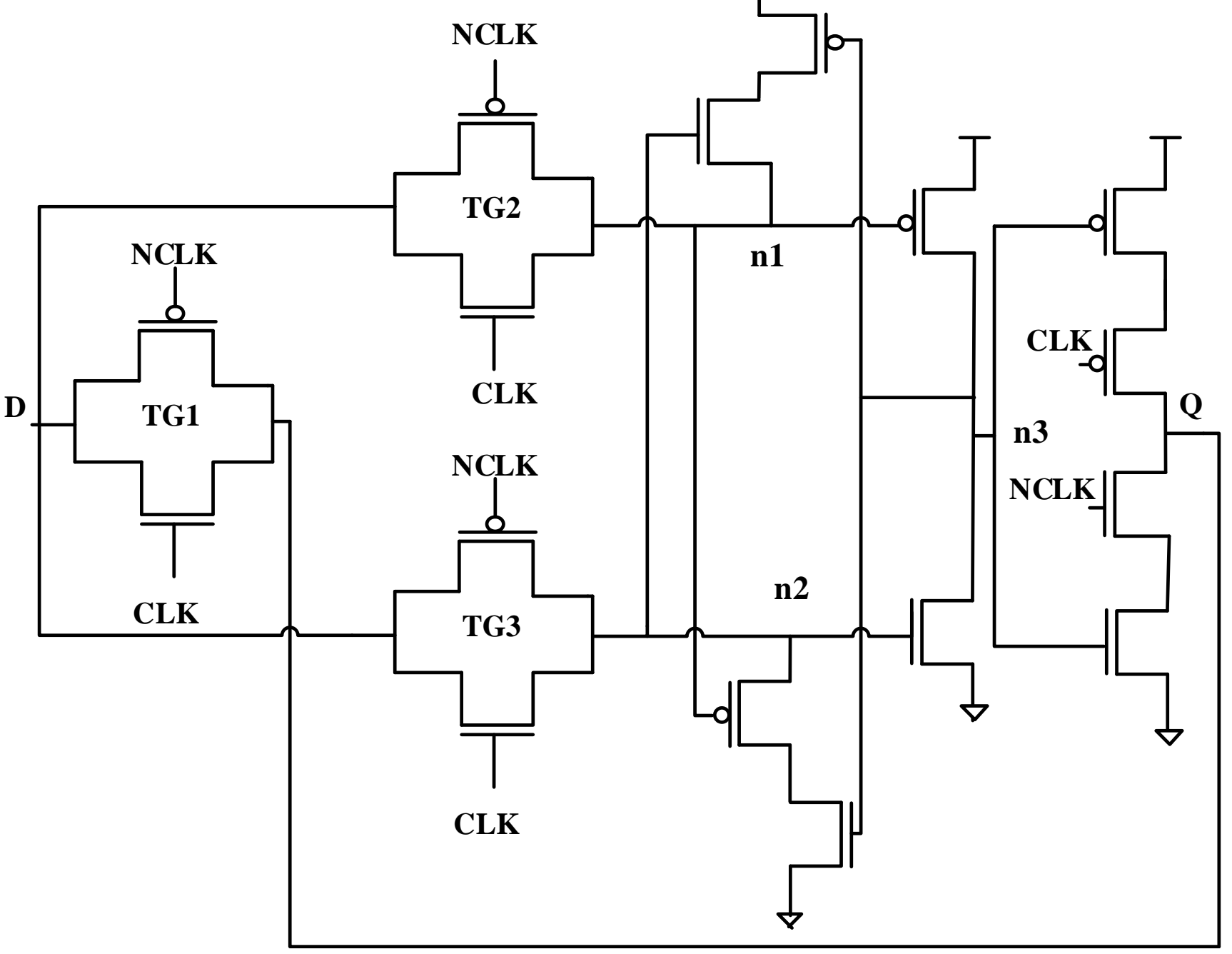

Fig. 7. Latch in [11]. 


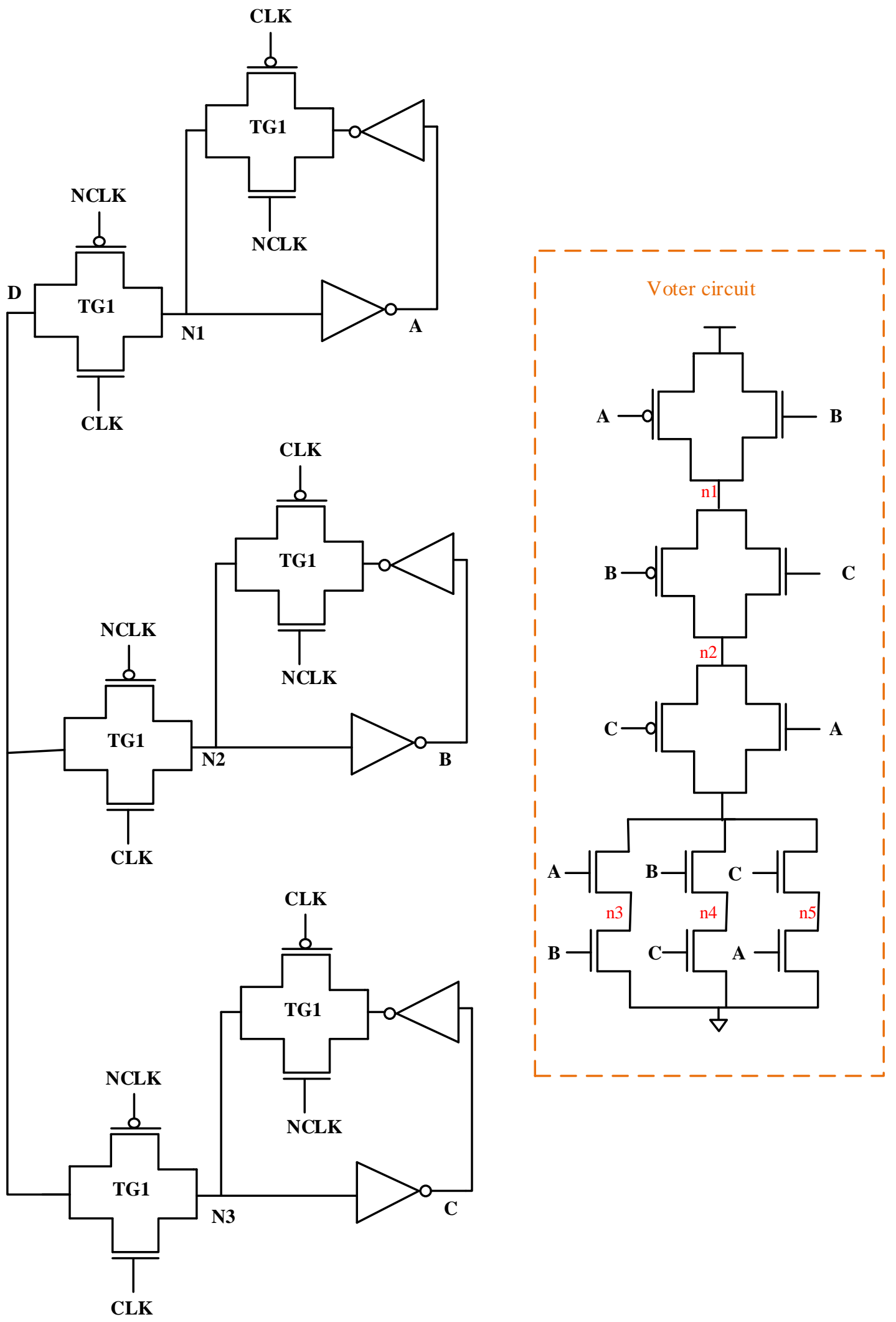

Fig. 8. Classical TMR Latch. 


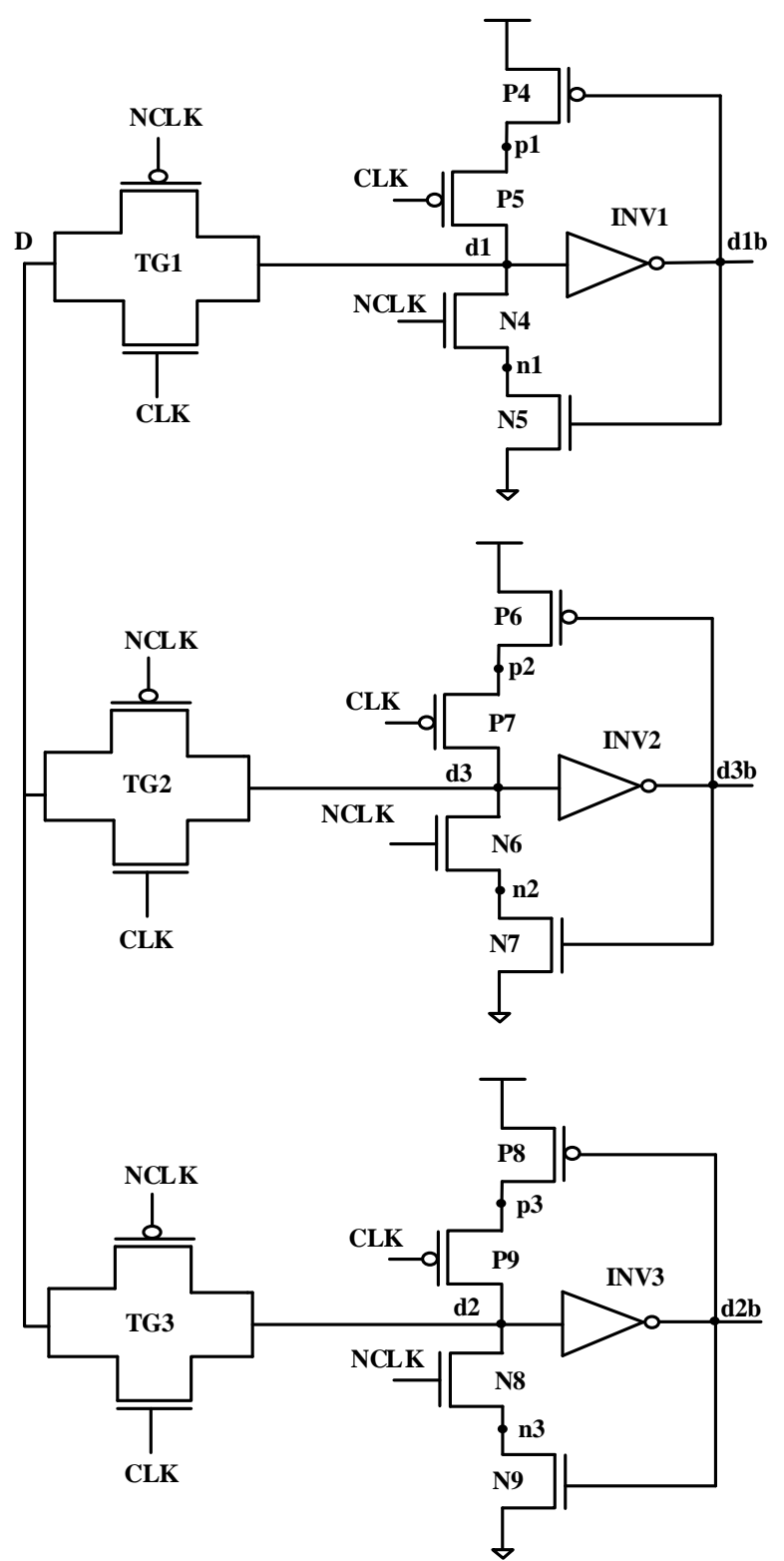

(a)

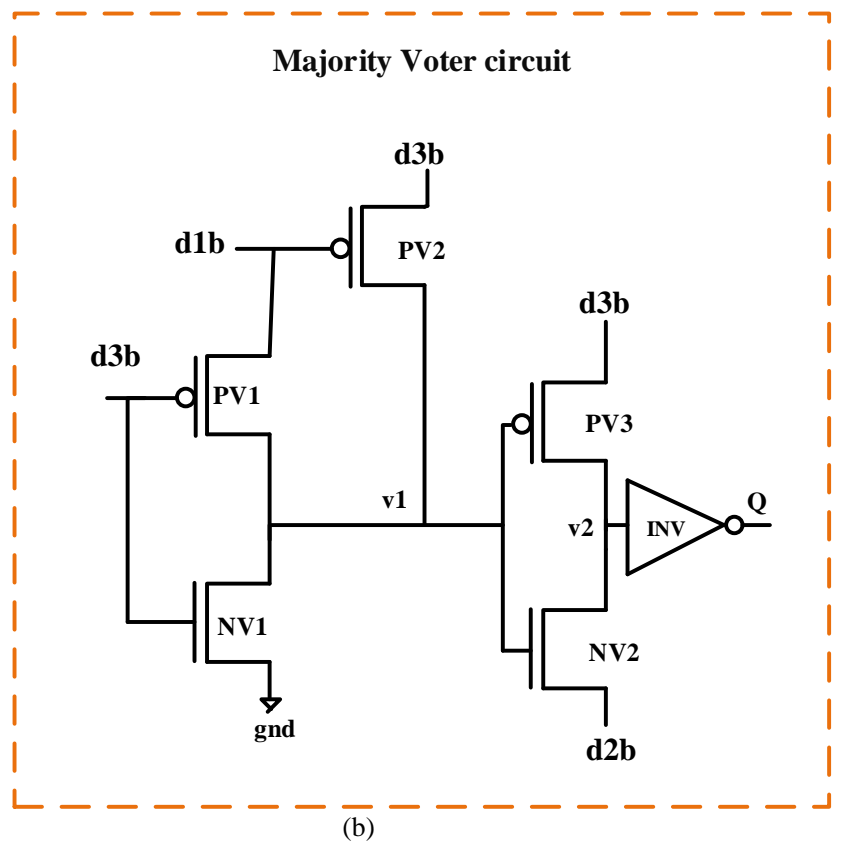

Fig. 9. Proposed FRTMR Latch: (a) Three Identical Latch Configurations; (b) Majority Voter Circuit.

\section{PROPOSED FRTMR HARDENED LATCH}

The proposed FRTMR latch is shown in Fig. 9. From Fig. 9 , it can be observed that the three identical latch circuits shown in Fig. 9(a) perform the operation and the results are processed by a majority voter circuit shown in Fig. 9(b) to produce a solitary output. According to Fig. 9, D, Q, CLK and NCLK are input, output, system clock and system negative clock respectively.

During transparent mode (when CLK $=1 \&$ NCLK $=0$ ) the transmission gates (TG1 $-\mathrm{TG} 3$ ) are $\mathrm{ON}$. For $\mathrm{D}=0$, (i.e., $\mathrm{d} 1=$ $\mathrm{d} 3=\mathrm{d} 2=0$ ) the PMOS transistors of INV1 - INV3 are ON which results in $d 1 b=d 3 b=b 2 b=1$ and subsequently, these values are fed to majority voter circuit. In the majority voter circuit for $\mathrm{d} 1 \mathrm{~b}=\mathrm{d} 3 \mathrm{~b}=\mathrm{d} 2 \mathrm{~b}=1$, transistors PV1 and PV2 are $\mathrm{OFF}, \mathrm{NV} 1$ is $\mathrm{ON}$ and hence, $\mathrm{V} 1=0$. Consequently, node $\mathrm{v} 2=$
1 through PV3 transistor. This v2 node voltage propagates through inverter (INV) and produces the output $\mathrm{Q}=0$.

For $\mathrm{D}=1$, (i.e., $\mathrm{d} 1=\mathrm{d} 3=\mathrm{d} 2=1$ ) the NMOS transistors of INV1 - INV3 are ON which results in $d 1 b=d 3 b=d 2 b=0$ and subsequently, these values are fed to majority voter circuit and makes $\mathrm{v} 1=0$. As a result, node $\mathrm{v} 2=0$ and hence, output $\mathrm{Q}=1$.

During latch mode (when CLK $=0 \&$ NCLK =1) the transmission gates (TG1 - TG3) are OFF, and hence, the internal nodes retain their current values through the NMOS transistors of feedback loops for $\mathrm{D}=0$ and PMOS transistors of feedback loops for $\mathrm{D}=1$. As a result, the latch outputs the correct value in the latch mode.

Now, the detailed working of FRTMR latch in the presence of transient fault at various internal nodes $(\mathrm{d} 1, \mathrm{~d} 3, \mathrm{~d} 2, \mathrm{~d} 1 \mathrm{~b}, \mathrm{~d} 3 \mathrm{~b}$, $\mathrm{d} 2 \mathrm{~b}, \mathrm{v} 1$ and $\mathrm{v} 2)$ is explained in this section. Note that, the 
storage of 0 of the latch is considered for all fault-tolerance discussions throughout the paper. In normal operation (without transient fault) for data input $\mathrm{D}=0$ case, $\mathrm{d} 1=\mathrm{d} 3=\mathrm{d} 2=\mathrm{v} 1=\mathrm{Q}$ $=0$ and $\mathrm{d} 1 \mathrm{~b}=\mathrm{d} 3 \mathrm{~b}=\mathrm{d} 2 \mathrm{~b}=\mathrm{v} 2=1$. In general, SET is analyzed only in latch mode.

SET on node $\mathrm{d} 1$ : In this case, $\mathrm{d} 1$ flips its state from $0 \rightarrow 1$, and the corresponding node $\mathrm{d} 1 \mathrm{~b}$ changes its state from $1 \rightarrow 0$. Therefore, the node voltages at $\mathrm{d} 1 \mathrm{~b}, \mathrm{~d} 3 \mathrm{~b}$ and $\mathrm{d} 2 \mathrm{~b}$ are 0,1 and 1 respectively. These values are fed to majority voter circuit. In the majority voter circuit for $\mathrm{d} 1 \mathrm{~b}=0$ and $\mathrm{d} 3 \mathrm{~b}=\mathrm{d} 2 \mathrm{~b}=1$, transistor PV1 is OFF, PV2 and NV1 are ON simultaneously. In order to perform the latch operation correctly, PV2 transistor in the majority voter circuit shown in Fig. 9(b) is sized 10 times (i.e., W/L $=1500 \mathrm{~nm} / 45 \mathrm{~nm}$ ) faster than NV1 (i.e., W/L $=150 \mathrm{~nm} / 45 \mathrm{~nm})$. The sizing of these transistors helps in maintaining the value of $\mathrm{v} 1$ at logic 1 . As a result, the node $\mathrm{v} 2$ becomes 1 . This v2 node voltage drives the inverter (INV) and produces the correct output. The voltage levels at node v1 for various $\mathrm{W} / \mathrm{L}$ ratios of $\mathrm{PV} 2$ and $\mathrm{NV} 1$ for different combinations of $d 1 b, d 3 b$ and $d 2 b$ are shown in Fig. 10.

SET on node d3: In this case, d3 flips its state from $0 \rightarrow 1$, and the corresponding node $\mathrm{d} 3 \mathrm{~b}$ changes its state from $1 \rightarrow 0$. Therefore, the node voltages at $\mathrm{d} 1 \mathrm{~b}, \mathrm{~d} 3 \mathrm{~b}$ and $\mathrm{d} 2 \mathrm{~b}$ are 1,0 and 1 respectively. These values are fed to majority voter circuit. In the majority voter circuit for $\mathrm{d} 3 \mathrm{~b}=0$ and $\mathrm{d} 1 \mathrm{~b}=\mathrm{d} 2 \mathrm{~b}=1$, transistors NV1 and PV2 are OFF, PV1 is ON and hence, v1= 1 and successively, v2 $=1$. This v2 node voltage is propagated through inverter (INV) to produce the correct output.

SET on node d2: In this case, d2 flips its state from $0 \rightarrow 1$, and the corresponding node $\mathrm{d} 2 \mathrm{~b}$ changes its state from $1 \rightarrow 0$. Therefore, the node voltages at $\mathrm{d} 1 \mathrm{~b}, \mathrm{~d} 3 \mathrm{~b}$ and $\mathrm{d} 2 \mathrm{~b}$ are 1,1 and 0 respectively. These values are fed to majority voter circuit. In the majority voter circuit for $\mathrm{d} 2 \mathrm{~b}=0$ and $\mathrm{d} 1 \mathrm{~b}=\mathrm{d} 3 \mathrm{~b}=1$, transistors PV1 and PV2 are OFF, NV1 is ON and hence $\mathrm{v} 1=$ 0 and successively, v2 $=1$ through PV3 transistor. This v2 node voltage is propagated through inverter (INV) to produce the correct output. The analysis is same for $\mathrm{d} 1 \mathrm{~b}, \mathrm{~d} 3 \mathrm{~b}$ and $\mathrm{d} 2 \mathrm{~b}$.

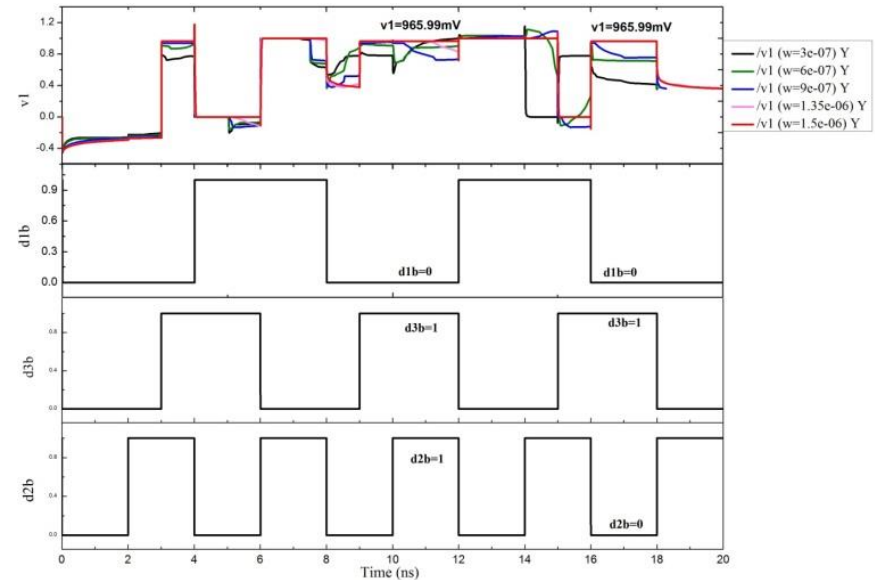

Fig. 10. Parametric Analysis to Determine the width of the Transistors $\mathrm{W}_{\mathrm{PV} 2}$ $/ \mathrm{W}_{\mathrm{NV} 1 . .}$
SET on node v1: Without transient fault for $D=0, d 1=\mathrm{d} 3$ $=\mathrm{d} 2=0, \mathrm{~d} 1 \mathrm{~b}=\mathrm{d} 3 \mathrm{~b}=\mathrm{d} 2 \mathrm{~b}=1$, and the internal node $\mathrm{v} 1$ and $\mathrm{v} 2$ are 0 and 1 respectively. Now, If transient fault occurs on node v1, it flips its state from $0 \rightarrow 1$. Subsequently, node v2 becomes 1 . This v2 node voltage drives the inverter (INV) and produces the correct output.

SET on node v2: If the transient fault occurs at node v2, it produces glitch at the output immediately and remains until the transient fault effect presents at the node. The output will be recovered after the transient fault dies down. In the proposed latch, internal nodes $\mathrm{d} 1, \mathrm{~d} 3, \mathrm{~d} 2, \mathrm{~d} 1 \mathrm{~b}, \mathrm{~d} 3 \mathrm{~b}, \mathrm{~d} 2 \mathrm{~b}$ and $\mathrm{v} 1$ are termed as "self-recoverable" nodes and v2 as "critical node".

Fig. 11 shows the simulation results of FRTMR latch for without and with transient fault injections (highlighted in the Fig. 11) on internal nodes $\mathrm{d} 1, \mathrm{~d} 3, \mathrm{~d} 2, \mathrm{~d} 1 \mathrm{~b}, \mathrm{~d} 3 \mathrm{~b}$ and $\mathrm{d} 2 \mathrm{~b}$ at different time periods. The minimum threshold current value imposed on the internal nodes is $40 \mu \mathrm{A}$. Similarly, Fig. 12 shows the simulation results of FRTMR latch with transient fault injections on nodes v1 and v2 at different time periods. The minimum threshold current value applied to these nodes is $60 \mu \mathrm{A}$. From Fig. 11 and Fig. 12, it can be observed that transient fault at any internal node (except v2) can be $100 \%$ tolerable since the output node $\mathrm{Q}$ constantly remains at correct value. In case of node v2, the output produces glitch and the glitch remains until the transient fault dies down.

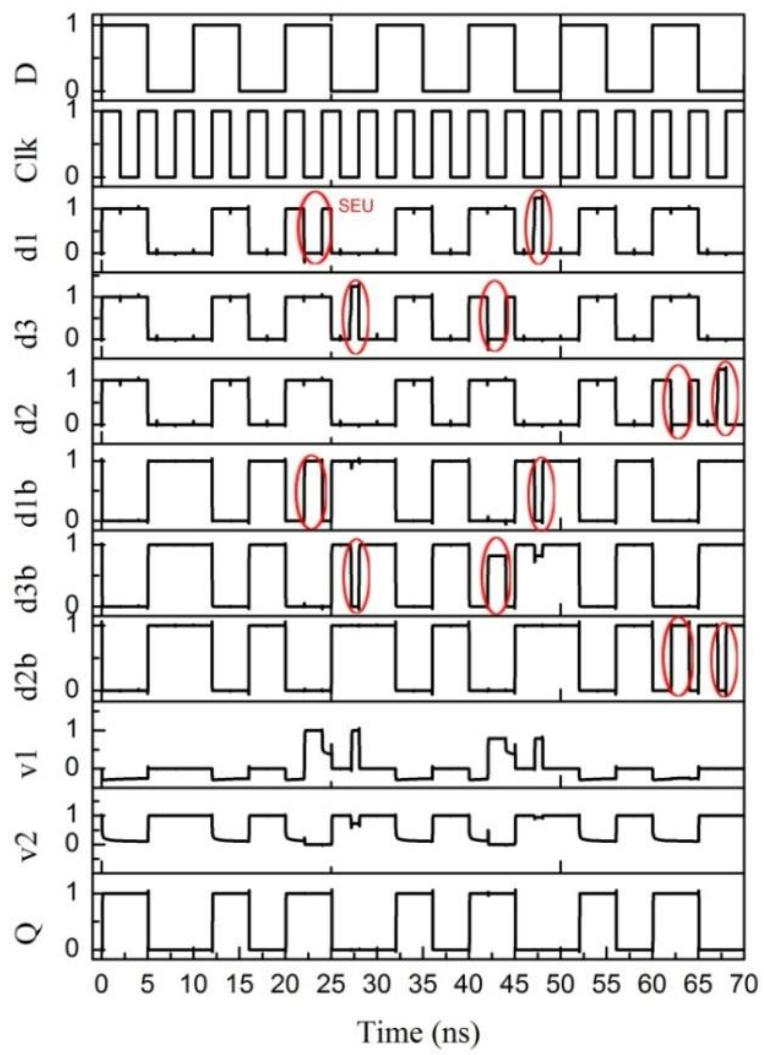

Fig. 11. Simulation Results of FRTMR latch without and with Transient Fault Injections at $\mathrm{d} 1, \mathrm{~d} 3, \mathrm{~d} 2$ for $\mathrm{D}=0$ \& 1 . 


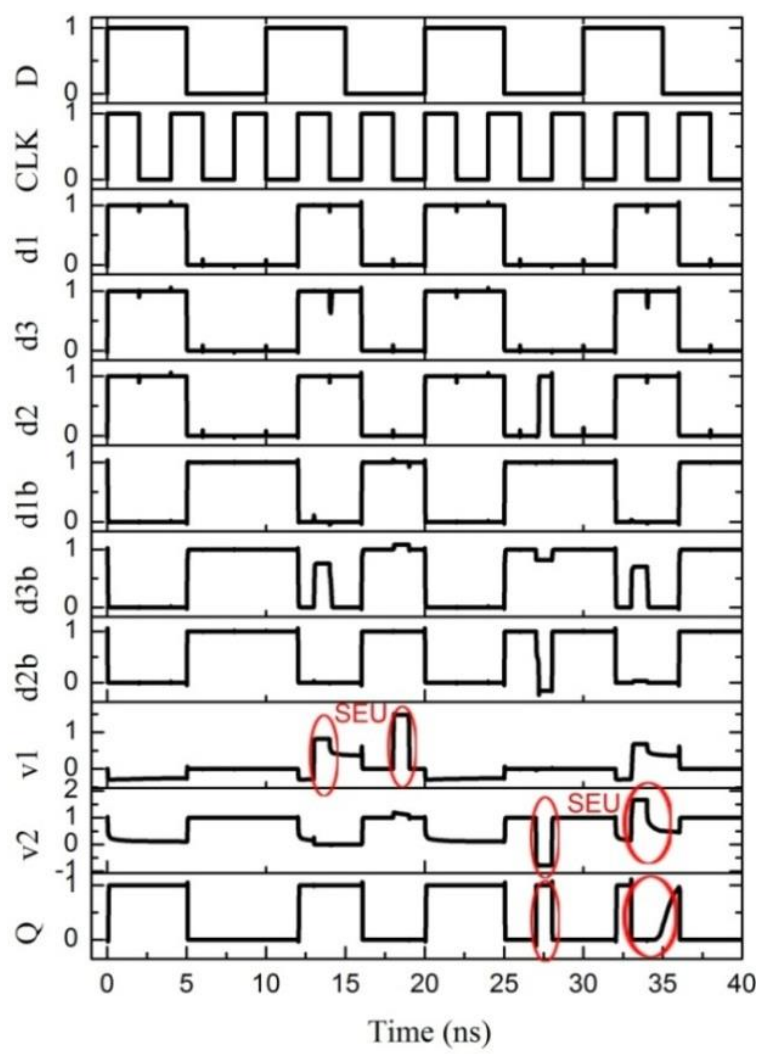

Fig. 12. Simulation Results of FRTMR latch with Transient Fault Injections at $\mathrm{v} 1$ and $\mathrm{v} 2$ for $\mathrm{D}=0$ and 1 .

From Fig. 11 and Fig. 12, statistics results for the SETs at internal nodes of FRTMR latch design are extracted and presented in Table I. According to Table I, for $\mathrm{D}=1$, transient fault is injected at $22 \mathrm{~ns}$ on node $\mathrm{d} 1$. It can be observed that the change in node voltage from $1 \rightarrow 0$, changes $\mathrm{d} 1 \mathrm{~b}$ from $0 \rightarrow 1$. Although, the output remains correct. Likewise, it is true for the other cases too. Table I also shows the statistics results for $\mathrm{D}=1$. From the Table I it can be observed that result "SR" denotes as "self-recoverable" nodes, where the output can completely self-recovered from any single node transient injection. Similarly "CN" as "critical nodes" where these nodes can flip the output node voltage and remains until the transient fault injection is removed from that node.

The detailed behaviour of FRTMR latch at internal nodes (d1, d3, d2, d1b, d3b, d2b, v1 and v2) for the data inputs D = 0 $\& 1$ is presented in Table II. Transient fault occurrence to the nodes is highlighted in the table.

\section{A. Multiple Event Transient Analysis of FRTMR Latch}

This section elaborates the multi-node upset tolerance analysis of the FRTMR latch. Multiple Event Transients (METs) may occur due to packing density or single events cause multiple transients. However, TMR circuits are only immune to SETs that effect to a single redundancy. Multiple SETs that affect multiple redundancies causes functional failure. Our definition of multi-node in this paper is a node on any one of the redundant latch and node v1 of voter circuit. Node v2 is not considering for multi node upsets as this is a critical node.
MET on $\langle$ d1, v1 >: d1 flip from $0 \rightarrow 1$ and d1b from $1 \rightarrow$ 0 , the nMOS transistor NV1 is ON and hence $v 1=0$. On the other hand, since $\mathrm{v} 1$ is also affected by transient fault simultaneously, it becomes 1 . Thus, v2 $=1$. Hence, output $\mathrm{Q}$ retains its correct value i.e., $\mathrm{Q}=0$.

MET on $\langle\mathbf{d} 3$, v1 $>$ : d3 flip from $0 \rightarrow 1$ and d3b from $1 \rightarrow$ 0 , the pMOS transistor PV1 is ON and hence, $v 1=1$. On the other hand, due to transient fault at v1simultaneously, v1 also tries to become 1 . This makes v $2=1$. Hence, output $\mathrm{Q}$ retains its correct value i.e., $\mathrm{Q}=0$.

MET on $\langle\mathbf{d} 2$, v1 $>$ : $\mathrm{d} 2$ flip from $0 \rightarrow 1$ and d2b from $1 \rightarrow$ 0 , the nMOS transistor NV1 is ON and hence, v1 = 0. But, due to transient fault at $\mathrm{v} 1$, it becomes 1 straight away. This makes $\mathrm{v} 2=0$. Resulting a wrong data stored at the output i.e., $\mathrm{Q}=1$. As discussed in the section II, this pair of node is treated as critical node.

Similarly, in the case of 1 being stored, it can be found through an examination that the MET on $\langle\mathrm{d} 1$, v1 $\rangle$ and $\langle\mathrm{d} 1$, v1 $>$ are $100 \%$ self-recoverable, whereas MET on $\langle\mathrm{d} 2$, v1 $>$ produces the wrong output. Fig. 13 shows the simulation results of METs of FRTMR latch for data inputs 0 and 1. From Fig. 13, statistics results for the METs at internal node pairs discussed above of FRTMR latch design are extracted and presented in Table III.

TABLE. I. STATISTICS RESUltS OF SET INJECTION OF FRTMRLATCH BASED ON FIG. $11 \& 12$ (FOR D = $1 \& 0$ )

\begin{tabular}{|l|l|l|l|l|l|l|l|}
\hline $\begin{array}{l}\text { Time } \\
(\mathbf{n s})\end{array}$ & Node & $\begin{array}{l}\text { (D= 1) } \\
\text { Output }\end{array}$ & Result & $\begin{array}{l}\text { Time } \\
(\mathbf{n s})\end{array}$ & Node & $\begin{array}{l}\text { (D = 0) } \\
\text { Output }\end{array}$ & Result \\
\hline 22 & $\mathrm{~d} 1$ & $\mathrm{Q}=1$ & $\mathrm{SR}$ & 47 & $\mathrm{~d} 1$ & $\mathrm{Q}=0$ & $\mathrm{SR}$ \\
\hline 42 & $\mathrm{~d} 3$ & $\mathrm{Q}=1$ & $\mathrm{SR}$ & 27 & $\mathrm{~d} 3$ & $\mathrm{Q}=0$ & $\mathrm{SR}$ \\
\hline 62 & $\mathrm{~d} 2$ & $\mathrm{Q}=1$ & $\mathrm{SR}$ & 67 & $\mathrm{~d} 2$ & $\mathrm{Q}=0$ & $\mathrm{SR}$ \\
\hline 73 & $\mathrm{v} 1$ & $\mathrm{Q}=1$ & $\mathrm{SR}$ & 93 & $\mathrm{v} 1$ & $\mathrm{Q}=0$ & $\mathrm{SR}$ \\
\hline 87 & $\mathrm{v} 2$ & $\mathrm{Q}=0$ & $\mathrm{CN}$ & 97 & $\mathrm{v} 2$ & $\mathrm{Q}=1$ & $\mathrm{CN}$ \\
\hline
\end{tabular}

TABLE. II. SEU ANALYSIS ON INTERNAL NODES WITH DATA INPUT (D = $0 \& 1)$

\begin{tabular}{|l|l|l|l|l|l|l|l|l|l|}
\hline $\begin{array}{l}\text { Input } \\
(\mathbf{D})\end{array}$ & $\mathbf{d 1}$ & $\mathbf{d 3}$ & $\mathbf{d 2}$ & $\mathbf{d} 1 \mathbf{b}$ & $\mathbf{d 3 b}$ & $\mathbf{d} 2 \mathbf{b}$ & $\mathbf{v 1}$ & $\mathbf{v 2}$ & $\begin{array}{l}\text { Output } \\
(\mathbf{Q})\end{array}$ \\
\hline 0 & 0 & 0 & 0 & 1 & 1 & 1 & 0 & 1 & $\begin{array}{l}0 \text { (without } \\
\text { SEU) }\end{array}$ \\
\hline 0 & $\mathbf{1}$ & 0 & 0 & 0 & 1 & 1 & 1 & 1 & $\sqrt{ }$ \\
\hline 0 & 0 & $\mathbf{1}$ & 0 & 1 & 0 & 1 & 1 & 1 & $\sqrt{ }$ \\
\hline 0 & 0 & 0 & $\mathbf{1}$ & 1 & 1 & 0 & 0 & 1 & $\sqrt{ }$ \\
\hline 0 & 0 & 0 & 0 & 1 & 1 & 1 & $\mathbf{1}$ & 1 & $\sqrt{ }$ \\
\hline 0 & 0 & 0 & 0 & 1 & 1 & 1 & 0 & $\mathbf{0}$ & $\times$ \\
\hline 1 & 1 & 1 & 1 & 0 & 0 & 0 & 0 & 0 & $\begin{array}{l}\text { SEU) } \\
\text { SEUthout }\end{array}$ \\
\hline 1 & $\mathbf{0}$ & 1 & 1 & 1 & 0 & 0 & 1 & 0 & $\sqrt{ }$ \\
\hline 1 & 1 & $\mathbf{0}$ & 1 & 0 & 1 & 0 & 1 & 0 & $\sqrt{ }$ \\
\hline 1 & 1 & 1 & $\mathbf{0}$ & 0 & 0 & 1 & 0 & 0 & $\sqrt{ }$ \\
\hline 1 & 1 & 1 & 1 & 0 & 1 & 0 & $\mathbf{1}$ & 0 & $\sqrt{ }$ \\
\hline 1 & 1 & 1 & 0 & 0 & 0 & 0 & 0 & $\mathbf{1}$ & $\times$ \\
\hline
\end{tabular}




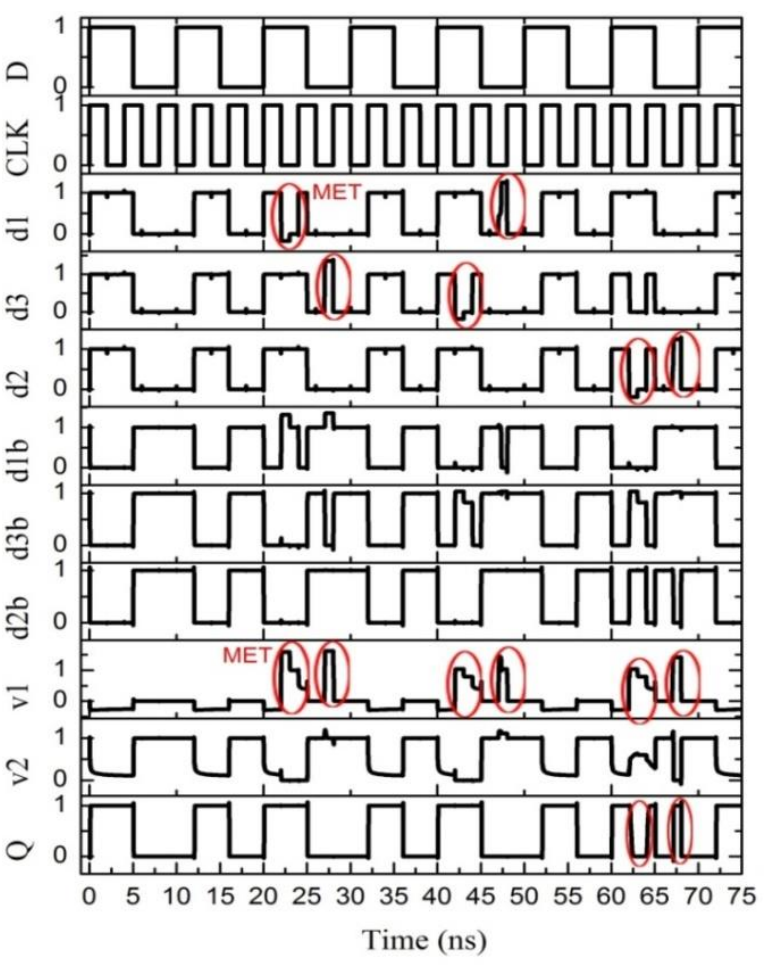

Fig. 13. Simulation Results of MET Injections of the FRTMR Latch for $D=0$ and 1.

TABLE. III. STATISTICS RESUlTS OF MET INJECTION OF FRTMR LATCH BASED ON FIG. 13 (FOR D $=1 \& 0$ )

\begin{tabular}{|l|l|l|l|l|l|l|l|}
\hline $\begin{array}{l}\text { Tim } \\
\mathbf{e} \\
(\mathbf{n s})\end{array}$ & Node & $\begin{array}{l}\text { Outpu } \\
\mathbf{t} \\
(\mathbf{D}=\mathbf{1})\end{array}$ & $\begin{array}{l}\text { Resul } \\
\mathbf{t}\end{array}$ & $\begin{array}{l}\text { Tim } \\
\mathbf{e} \\
(\mathbf{n s})\end{array}$ & Node & $\begin{array}{l}\text { Outpu } \\
\mathbf{t} \\
(\mathbf{D}=\mathbf{0})\end{array}$ & $\begin{array}{l}\text { Resul } \\
\mathbf{t}\end{array}$ \\
\hline 22 & $\begin{array}{l}<\mathrm{d} 1, \mathrm{v} 1 \\
>\end{array}$ & $\mathrm{Q}=1$ & $\mathrm{SR}$ & 47 & $\begin{array}{l}<\mathrm{d} 1, \mathrm{v} 1 \\
>\end{array}$ & $\mathrm{Q}=0$ & $\mathrm{SR}$ \\
\hline 42 & $\begin{array}{l}<\mathrm{d} 3, \mathrm{v} 1 \\
>\end{array}$ & $\mathrm{Q}=1$ & $\mathrm{SR}$ & 27 & $\begin{array}{l}<\mathrm{d} 3, \mathrm{v} 1 \\
>\end{array}$ & $\mathrm{Q}=0$ & $\mathrm{SR}$ \\
\hline 62 & $\begin{array}{l}<\mathrm{d} 2, \mathrm{v} 1 \\
>\end{array}$ & $\mathrm{Q}=0$ & $\mathrm{CN}$ & 67 & $\begin{array}{l}<\mathrm{d} 2, \mathrm{v} 1 \\
>\end{array}$ & $\mathrm{Q}=1$ & $\mathrm{CN}$ \\
\hline
\end{tabular}

\section{B. Novel Majority Voter Circuit}

Majority voter circuit plays a vital role in TMR design. In TMR circuits, voter circuits are placed around redundant latches to continuously monitor the three logic redundancies. When an SET occurs on any one of the three redundancies, these voter circuits prevent the latches from permanent failure of the logic. The output expression of a transistor level majority voter circuit from Fig. 8 can be derived as:

$((\mathrm{A} \times \mathrm{B})+(\mathrm{B} \times \mathrm{C})+(\mathrm{C} \times \mathrm{A}))^{\prime}$

As per the discussion in section II, the disadvantage of the majority voter circuit is, it has more number of critical nodes (n1-n5) i.e., 5 critical nodes. The critical nodes are inversely proportional to reliability. This paper proposes a novel majority voter circuit with only 2 internal nodes out of which, 1 is signified as self-recoverable node and the other is signified as critical node. Equation (2), (3) and (4) are derived at internal nodes v1, v2 and Q of majority voter circuit shown in Fig. 9(b).
$\mathrm{V} 1=\mathrm{d} 1 \mathrm{~b} \oplus \mathrm{d} 3 \mathrm{~b}$

$\mathrm{V} 2=[(\mathrm{d} 1 \mathrm{~b} \times \mathrm{d} 3 \mathrm{~b})+\mathrm{d} 2 \mathrm{~b}(\mathrm{~d} 1 \mathrm{~b} \oplus \mathrm{d} 3 \mathrm{~b})]$

$Q=[(d 1 b \times d 3 b)+d 2 b(d 1 b \oplus d 3 b)]^{\prime}$

\section{PeRformance EVAluation ANd COMPaRison}

A classical TMR latch shown in Fig. 8 is considered as reference latch as this circuit is also used a voter circuit for hardening technique. To examine the performance of FRTMR latch, delay $(\mathrm{D} \rightarrow \mathrm{Q})$, power, area, PDP and critical nodes are considered. For the purpose of comparison, the proposed FRTMR latch and existing latches, namely, LCHR, REFL, Latch in [9], [10], [11] and classical TMR Latch are implemented in $45 \mathrm{~nm}$ technology with the supply voltage of $1 \mathrm{~V}$ and $250 \mathrm{MHz}$ clock frequency. The implemented latches are simulated in Cadence spectre environment. Table IV compares the evaluation costs for the FRTMR and existing latches with regard to delay, power, PDP, area, critical nodes, etc. From the Table IV, it may be noted that the power consumption of FRTMR latch is $85 \%, 44.3 \%, 88 \%$ and $17.83 \%$ reduced compared to LCHR, Latch in [9], Latch in [10] and classical TMR latch. And also it is third lowest compared with the latches reported in the Table IV. FRTMR latch has 13.8\%, $18.4 \%$ and $13.88 \%$ reduction in area when compared to LCHR, Latch in [10] and classical TMR latch. But it has a trade-off in delay compared to reference latch. It is only best to LCHR latch. Nevertheless, FRTMR latch is more robust for SETs because of the less number of critical nodes (only 1). The proposed FRTMR latch can tolerate multi-node upsets compared to all the latches reported in Table IV.

TABLE. IV. PERFORMANCE COMPARISONS

\begin{tabular}{|l|l|l|l|l|l|l|}
\hline & $\begin{array}{l}\text { Delay } \\
\text { (ps) }\end{array}$ & $\begin{array}{l}\text { Power } \\
(\mathbf{n W})\end{array}$ & $\begin{array}{l}\text { PDP } \\
\text { (fJ) }\end{array}$ & $\begin{array}{l}\text { Area } \\
\text { (\# of } \\
\text { transisto } \\
\text { rs) }\end{array}$ & $\begin{array}{l}\text { \# of } \\
\text { Critic } \\
\text { al } \\
\text { nodes }\end{array}$ & $\begin{array}{l}\text { MET } \\
\text { tolerant } \\
\text { (Yes/ } \\
\text { No) }\end{array}$ \\
\hline $\begin{array}{l}\text { LCHR in } \\
{[7]}\end{array}$ & 118.7 & 4880 & 580 & 36 & 7 & No \\
\hline $\begin{array}{l}\text { REFL in } \\
{[8]}\end{array}$ & 67 & 490 & 33 & 26 & 7 & No \\
\hline $\begin{array}{l}\text { Latch in } \\
{[9]}\end{array}$ & 26.38 & 1273 & 33.6 & 20 & - & No \\
\hline $\begin{array}{l}\text { Latch in } \\
{[10]}\end{array}$ & 73 & 6077 & 444 & 38 & 11 & No \\
\hline $\begin{array}{l}\text { Latch in } \\
{[11]}\end{array}$ & 3.99 & 211.3 & $\begin{array}{l}0.84 \\
1\end{array}$ & 16 & 2 & No \\
\hline $\begin{array}{l}\text { Classical } \\
\text { TMR latch }\end{array}$ & 48.16 & 863 & 41.6 & 36 & 5 & No \\
\hline $\begin{array}{l}\text { FRTMR } \\
\text { latch }\end{array}$ & 66.76 & 709.09 & 47.3 & 31 & 1 & Yes \\
\hline
\end{tabular}

\section{CONCLUSION}

At deep sub-micron technology, the CMOS integrated circuits are more likely to experience the occurrence of SETs and METs. This paper proposed FRTMR latch with novel majority voter circuit in $45 \mathrm{~nm}$ technology which can tolerate single and multi-node upsets. Generally, the TMR structures consume large silicon area and high power consumption. But, 
the proposed FRTMR latch offers, low power and less area i.e. $17.83 \%$ and $13.88 \%$ respectively compared to existing classical TMR latch. The simulation results demonstrate that the FRTMR latch has $80 \%$ improved SEU tolerance than the classical TMR latch. Nonetheless, it has a tradeoff in delay i.e., $38.6 \%$ more delay compared to classical TMR latch.

\section{REFERENCES}

[1] Baumann, Robert, "Soft errors in advanced computer systems," IEEE Design \& Test of Computers, vol.22, no. 3, pp. 258-266, 2005.

[2] Ferlet-Cavrois, Veronique and Massengill, Lloyd W and Gouker, Pascale, "Single event transients in digital CMOS - A review," IEEE Trans. Nuclear Science, vol.60, no. 3, pp. 1767-1790, 2013.

[3] Saremi, Mehdi and Privat, Aymeric and Barnaby, Hugh J and Clark, Lawrence T, "Physically based predictive model for single event transients in CMOS gates," IEEE Trans. Electron Devices, vol. 63, no. 6, pp. 2248-2254, 2016.

[4] Black, Dolores A and Robinson, William H and Wilcox, Ian Z and Limbrick, Daniel B and Black, Jeffrey D, "Modelling of single event transients with dual double-exponential current sources: Implications for logic cell characterization," IEEE Trans. Nuclear Science, vol.62, no. 4, pp. 1540-1549, 2015.

[5] Aketi, Sai Aparna and Mekie, Joycee and Shah, Hemal, "Single-error hardened and multiple-error tolerant guarded dual modular redundancy technique," 2018 31st International Conference on VLSI Design and 2018 17th International Conference on Embedded Systems (VLSID), pp. 250-255, 2018.

[6] Yan, Aibin and Huang, Zhengfeng and Fang, Xiangsheng and Ouyang,Yiming and Deng, Honghui, "Single event double-upset fully immune and transient pulse filterable latch design for nanoscale CMOS," Microelectronics Reliability, vol.61, pp. 43-50, 2017.

[7] Qi, C., Xiao, L., Guo, J. and Wang, T. "Low cost and highly reliable radiation hardened latch design in $65 \mathrm{~nm}$ CMOS technology", Microelectronics Reliability, 55(6), pp.863-872, 2015.

[8] Yan, A., Liang, H., Huang, Z., Jiang, C., Ouyang, Y. and Li, X., "An SEU resilient, SET filterable and cost effective latch in presence of PVT variations. Microelectronics Reliability, 63, pp.239-250, 2016.

[9] Liu, P., Zhao, T., Liang, F., Zhao, J. and Jiang, P. "A power-delayproduct efficient and SEU-tolerant latch design", IEICE Electronics Express, 14(23), pp.20170972-20170972, 2017.
[10] Xu, H., Zhu, J., Lu, X. and Li, J. "An advanced SEU tolerant latch based on error detection", Journal of Semiconductors, 39(5), p.055003, 2018.

[11] Kumar, C.I. and Bulusu, A. "High performance energy efficient radiation hardened latch for low voltage applications", Integration 2019.

[12] Huang, Z., Liang, H. and Hellebrand, S. "A high performance SEU tolerant latch", Journal of Electronic Testing, 31(4), pp.349-359, 2015.

[13] Zhengfeng, H. and Huaguo, L., "A novel radiation hardened by design latch", Journal of Semiconductors, 30(3), p.035007, 2009.

[14] Ramamurthy, C., Chellappa, S., Vashishtha, V., Gogulamudi, A. and Clark, L.T. "High performance low power pulse-clocked TMR circuits for soft-error hardness", IEEE Transactions on Nuclear Science, 62(6), pp.3040-3048, 2015.

[15] Nan, H. and Choi, K. "Novel radiation hardened latch design considering process, voltage and temperature variations for nanoscale MOS technology", Microelectronics Reliability, 51(12), pp.2086-2092, 2011.

[16] Gadlage, M.J., Ahlbin, J.R., Gadfort, P., Roach, A.H. and Stansberry, S., "Characterization of single-event transients in Schmitt trigger inverter chains operating at subthreshold voltages", IEEE Transactions on Nuclear Science, 64(1), pp.637-642, 2016.

[17] Lin, S., Kim, Y.B. and Lombardi, F. "Design and performance evaluation of radiation hardened latches for nanoscale CMOS", IEEE Transactions on Very Large Scale Integration (VLSI) Systems, 19(7), pp.1315-1319, 2010.

[18] Nan, H. and Choi, K., "Low cost and highly reliable hardened latch design for nanoscale CMOS technology", Microelectronics Reliability, 52(6), pp.1209-1214, 2012.

[19] Rajaei, R., Tabandeh, M. and Fazeli, M., "Low cost soft error hardened latch designs for nano-scale CMOS technology in presence of process variation”, Microelectronics Reliability, 53(6), pp.912-924, 2013.

[20] Jagannathan, S., Loveless, T.D., Bhuva, B.L., Wen, S.J., Wong, R., Sachdev, M., Rennie, D. and Massengill, L.W. "Single-event tolerant flip-flop design in 40-nm bulk CMOS technology", IEEE Transactions on Nuclear Science, 58(6), pp.3033-3037, 2011.

[21] Liu, Xin. "Multiple Node Upset-Tolerant Latch Design." IEEE Transactions on Device and Materials Reliability, 2019.

[22] Ding, Lili, Wei Chen, Tan Wang, Rongmei Chen, Yinhong Luo, Fengqi Zhang, Xiaoyu Pan, Huabo Sun, and Lei Chen. "Modeling the dependence of single event transients on strike location for circuit-level simulation." IEEE Transactions on Nuclear Science, 2019. 